\title{
Evidence-Based PET for Haematological Tumours
}

\author{
Francesco Bertagna, Raffaele Giubbini, \\ and Domenico Albano
}

\subsection{Introduction}

Haematological malignancies include lymphomas such as Hodgkin lymphoma (HL) and nonHodgkin lymphoma (NHL), leukaemia and multiple myeloma (MM). They can affect any organ system and positron emission tomography/ computed tomography (PET/CT) has been accepted as part of the routine management of most of them. In this chapter were only considered recent systematic reviews and meta-analyses concerning the use of PET or PET/CT with ${ }^{18} \mathrm{~F}$ FDG in haematological malignancies dividing the results by the main areas of application.

\section{$9.2 \quad{ }^{18}$ F-FDG PET or PET/CT in Staging or Detection}

In staging HL and more aggressive NHL subtypes, ${ }^{18}$ F-FDG PET/CT was shown to be clearly more accurate than conventional radiological imaging to detect nodal and extranodal involvement. On the other hand, recent meta-analyses have addressed the diagnostic performance of this imaging method in some types of NHL, in $\mathrm{MM}$ and in the assessment of bone marrow involvement of HL and NHL [1-9].

F. Bertagna $(\varangle) \cdot$ R. Giubbini $\cdot$ D. Albano

Nuclear Medicine, University of Brescia and Spedali

Civili di Brescia, Brescia, Italy

e-mail: francesco.bertagna@unibs.it

\subsubsection{Post-transplant Lymphoproliferative Disorder}

Montes de Jesus et al. [1] evaluated the performance of advanced imaging modalities at diagnosis for post-transplant lymphoproliferative disorder (PTLD) after solid organ and haematopoietic stem cell transplantation. ${ }^{18} \mathrm{~F}-\mathrm{FDG}$ PET/ CT was the primary imaging modality investigated. Subgroup analysis of imaging results for detection and staging in patients with PTLD indicated that ${ }^{18} \mathrm{~F}$-FDG PET/CT identified additional lesions not detected by conventional imaging in $27.8 \%$ of cases, from which extranodal sites in $23.6 \%$. False negative results occurred in $11.5 \%$ of cases, predominantly in physiological high background activity regions and in early PTLD lesions. False positive results occurred in $4.8 \%$ of cases, predominantly due to inflammatory conditions. They concluded that ${ }^{18} \mathrm{~F}-\mathrm{FDG}$ $\mathrm{PET} / \mathrm{CT}$ is currently the most frequently investigated imaging modality in PTLD patients with promising results in detection and staging, but available studies suffer from methodological shortcomings.

\subsubsection{Follicular Lymphoma}

Adams et al. [2] studied the additional value of ${ }^{18}$ F-FDG PET to CT for staging newly diagnosed follicular lymphoma (FL) in terms of Ann Arbor 
staging and Follicular Lymphoma International Prognostic Index (FLIPI) risk stratification. The proportion of patients who were upstaged by ${ }^{18} \mathrm{~F}$ FDG PET compared with CT ranged from 0 to $45.2 \%$, with a pooled summary proportion of $18.7 \%$ (95\% confidence interval $(95 \% \mathrm{CI}): 10.8-$ $30.4 \%$ ). The single study that only included patients with CT-based limited non-bulky stage I to II disease reported ${ }^{18} \mathrm{~F}-\mathrm{FDG}$ PET-induced upstaging in $40.5 \%$ of cases. No study reported data on the influence of ${ }^{18} \mathrm{~F}$-FDG PET on FLIPI risk stratification. Although upstaging by ${ }^{18} \mathrm{~F}-$ FDG PET compared with CT occurs in a considerable proportion of patients, the available studies on this topic had numerous methodological errors. The authors concluded that future welldesigned studies are needed before ${ }^{18} \mathrm{~F}$-FDG PET can be recommended for routine pre-treatment staging of FL.

\subsubsection{Marginal Zone Lymphoma of the Mucosa-Associated Lymphoid Tissue}

Treglia et al. [3] analysed the detection rate (DR) of ${ }^{18} \mathrm{~F}-\mathrm{FDG}$ PET and PET/CT for the evaluation of patients with marginal zone lymphoma of the mucosa-associated lymphoid tissue (MALT). The pooled DR of ${ }^{18} \mathrm{~F}-\mathrm{FDG}$ PET or PET/CT was $71 \%$ (95\%CI: $61-80 \%$ ). A significant difference between the DR of PET/ CT (69\%; 95\%CI: $61-80 \%)$ and that of PET alone (73\%; 95\% CI: 60-84\%) was not demonstrated. A better DR of ${ }^{18} \mathrm{~F}-\mathrm{FDG}$ PET or PET/ CT in bronchial (94\%; 95\%CI: 85-99\%) and head-and-neck (90\%; 95\%CI: 78-98\%) MALT lymphomas compared with gastric (62\%; 95\%CI: 46-77\%) and ocular (49\%; 95\%CI: 36-63\%) MALT lymphomas was found. This meta-analysis demonstrated that MALT lymphoma is an ${ }^{18} \mathrm{~F}$-FDG-avid tumour in most of the cases, suggesting a potential clinical role in the initial evaluation of these patients. In particular, the DR of ${ }^{18} \mathrm{~F}$-FDG PET or PET/CT is related to the primary site of the MALT lymphoma.

\subsubsection{Bone Marrow Involvement in Lymphoma}

Adams et al. [4] analysed the diagnostic performance of ${ }^{18} \mathrm{~F}-\mathrm{FDG}$ PET/CT in detecting bone marrow involvement (BMI) in patients with newly diagnosed diffuse large B-cell lymphoma (DLBCL). The pooled sensitivity and specificity of ${ }^{18} \mathrm{~F}-\mathrm{FDG}$ PET/CT for detecting BMI were $88.7 \%$ (95\%CI: 82.5-93.3\%) and 99.8\% (95\% CI: 98.8-100\%), respectively. The area under the summary ROC curve was 0.9983 . They concluded that ${ }^{18} \mathrm{~F}-\mathrm{FDG}$ PET/CT is accurate and complementary to bone marrow biopsy (BMB) for detecting BMI in patients with newly diagnosed DLBCL. A negative ${ }^{18} \mathrm{~F}-\mathrm{FDG}$ PET/CT cannot rule out the presence of BMI, but positive ${ }^{18} \mathrm{~F}-\mathrm{FDG}$ PET/CT findings obviate the need for $\mathrm{BMB}$ for the detection of BMI in these patients.

The same group of authors systematically reviewed and meta-analysed published data on the diagnostic performance of ${ }^{18} \mathrm{~F}-\mathrm{FDG}$ PET/CT in detecting BMI in newly diagnosed HL to assess whether ${ }^{18} \mathrm{~F}-\mathrm{FDG}$ PET/CT can replace blind BMB in these patients [5]. The pooled sensitivity and specificity of ${ }^{18} \mathrm{~F}-\mathrm{FDG}$ PET/CT for the detection of BMI range were $96.9 \%$ (95\% CI: 93-99\%) and $99.7 \%$ (95\%CI: $98.9-100 \%)$, respectively. The area under the ROC curve was 0.986. In conclusion, although the methodological quality of studies that were included in this systematic review and meta-analysis was moderate, the meta-analysis suggests that ${ }^{18} \mathrm{~F}-\mathrm{FDG}$ $\mathrm{PET} / \mathrm{CT}$ may be an appropriate method to replace BMB in newly diagnosed HL.

Cheng et al. [6] also carried out a metaanalysis to evaluate the performance of ${ }^{18} \mathrm{~F}-\mathrm{FDG}$ PET and PET/CT against BMB in the initial diagnosis of BMI in patients with HL. Both ${ }^{18} \mathrm{~F}$ FDG PET and BMB had excellent specificity in detecting BMI. However, ${ }^{18} \mathrm{~F}-\mathrm{FDG}$ PET had excellent pooled sensitivity $(94.5 \%$; 95\% CI: 89.0-97.8\%) in detecting BMI in the initial staging of HL patients, whereas the pooled sensitivity of iliac BMB was very poor $(39.4 \%$; $95 \% \mathrm{CI}$ : $30.8-48.4 \%$ ). The authors concluded that ${ }^{18} \mathrm{~F}-$ FDG PET significantly outperforms iliac BMB in 
the detection of BMI in the initial staging of HL patients and therefore should be used as a firstline study.

\subsubsection{Natural Killer/T-Cell Lymphoma}

Ji et al. [7] evaluated the values of ${ }^{18} \mathrm{~F}-\mathrm{FDG}$ PET/ $\mathrm{CT}$ and PET in diagnosing extranodal nasal type natural killer/T-cell lymphoma (ENKTL). Pooled sensitivity, specificity and area under the curve (AUC) of ${ }^{18} \mathrm{~F}-\mathrm{FDG}$ PET/CT for diagnosing ENKTL were 97\% (95\%CI: 93-99\%), 97\% (95\%CI: 88-99\%) and 0.99 (95\%CI: 0.98-1.00). The same parameters for ${ }^{18} \mathrm{~F}-\mathrm{FDG}$ PET were 81\% (95\%CI: 70-89\%), 90\% (95\%CI: 66-98) and 0.86 (95\%CI: 0.82-0.89), respectively. The authors concluded that in comparison with PET, ${ }^{18}$ F-FDG PET/CT had excellent diagnostic value in detecting and staging ENKTL.

Zhou et al. [8] evaluated the role of ${ }^{18} \mathrm{~F}-\mathrm{FDG}$ $\mathrm{PET} / \mathrm{CT}$ in the diagnosis and staging of natural killer/T-cell lymphoma (NKTL). On a patientbased analysis, the pooled sensitivity and specificity of ${ }^{18} \mathrm{~F}-\mathrm{FDG}$ PET/CT in the diagnosis of NKTL were 95\% (95\%CI: 89-98\%) and 40\% (95\%CI: 9-78\%), respectively. For lesion-based analysis, the pooled sensitivity and specificity of ${ }^{18} \mathrm{~F}-\mathrm{FDG}$ PET/CT in the staging of NKTL were 98\% (95\%CI: 96-99\%) and 99\% (95\%CI: 99-100), respectively. The results indicated that ${ }^{18} \mathrm{~F}$-FDG PET/CT could be used as a valuable diagnostic and staging tool for NKTL.

\subsubsection{Multiple Myeloma}

Lu et al. [9] conducted a systematic review and meta-analysis to evaluate the diagnostic accuracy of ${ }^{18} \mathrm{~F}$-FDG PET or PET/CT for intramedullary and extramedullary lesions in MM. The pooled sensitivity and specificity of ${ }^{18} \mathrm{~F}$-FDG PET or PET/CT for the detection of extramedullary lesions in MM were 96.0\% (95\%CI: 79.6-99.9\%) and $77.8 \%$ (95\% CI: 40-97.2\%), respectively. The pooled sensitivity and specificity of ${ }^{18} \mathrm{~F}-\mathrm{FDG}$ PET or PET/CT for the detection of intramedul- lary lesions in $\mathrm{MM}$ were $61.1 \%$ (95\% CI: 43.5$76.9 \%$ ) and $94.1 \%$ (95\% CI: 71.3-99.9\%), respectively. They concluded that whole-body ${ }^{18} \mathrm{~F}-\mathrm{FDG}$ PET or PET/CT is a valuable imaging tool for the assessment of patients with MM, especially for the appraisal of extramedullary involvement.

\subsection{F-FDG PET or PET/CT in Treatment Response Evaluation (Interim and/or End of Therapy)}

\subsubsection{Post-transplant Lymphoproliferative Disorder}

In the meta-analysis by Montes de Jesus et al. [1] on imaging modalities in PTLD, the subgroup analysis of imaging results at treatment response evaluation indicated that ${ }^{18} \mathrm{~F}$-FDG PET/CT findings altered or guided treatment in $29 \%$ of cases. False positive results during treatment response evaluation were reported in $20 \%$ of cases, predominantly due to inflammatory conditions. They concluded that ${ }^{18} \mathrm{~F}$-FDG PET/CT may be promising in therapy evaluation but suffers from methodological shortcomings. Concerns remain with regard to occurrence of false negatives due to physiological high background activity and early PTLD lesions as well as false positives due to inflammatory conditions.

\subsubsection{Hodgkin and Non-Hodgkin Lymphomas}

Adams et al. [10] systematically reviewed and meta-analysed the proportion of false positive lesions at interim and end-of-treatment ${ }^{18} \mathrm{~F}-\mathrm{FDG}$ PET in lymphomas (both HL and NHL) using biopsy as reference standard. The pooled proportion of false positive results among all biopsied ${ }^{18}$ F-FDG-avid lesions at PET performed during or after completion of treatment was $55.7 \%$ (95\%CI: $32.6-76.6 \%)$. The pooled false positive proportions were $83 \%$ (95\% CI: $72-90.2 \%$ ) for interim ${ }^{18}$ F-FDG PET in NHL, $23.1 \%$ (95\%CI: 4.7-64.5\%) 
for end-of-treatment ${ }^{18} \mathrm{~F}-\mathrm{FDG}$ PET in $\mathrm{HL}$ and $31.5 \%$ (95\% CI: 3.9-83.9\%) for end-of-treatment ${ }^{18} \mathrm{~F}-\mathrm{FDG}$ PET in NHL. The authors concluded that both interim and end-of-treatment ${ }^{18} \mathrm{~F}$-FDG PET in patients with lymphoma suffer from a very high number of false positive findings.

Sun et al. [11] conducted a meta-analysis to evaluate the predictive value of interim ${ }^{18} \mathrm{~F}$-FDG PET/CT in patients with DLBCL treated with R-CHOP chemotherapy. The pooled sensitivity of interim ${ }^{18} \mathrm{~F}$-FDG PET/CT was $52.4 \%$ and the pooled specificity was $67.8 \%$. In conclusion, the sensitivity and specificity of interim ${ }^{18} \mathrm{~F}-\mathrm{FDG}$ PET/CT in predicting the outcome of DLBCL patients treated with R-CHOP chemotherapy were not satisfactory. To improve this, some more work should be done to unify the response criteria and some more research to assess the prognostic value of interim ${ }^{18} \mathrm{~F}$-FDG PET/CT with semi-quantitative analysis.

Ziakas et al. [12] assessed the diagnostic performance of interim ${ }^{18} \mathrm{~F}$-FDG PET with regard to the final outcome of adult patients with newly diagnosed HL. The pooled sensitivity was $67 \%$ (95\%CI: $57-76 \%$ ) and pooled specificity was 89\% (95\%CI: 84-93\%). The estimated negative predictive value was 93\% (95\%CI: $85-100 \%$ ). The diagnostic performance was influenced by most covariates tested, including age, duration of follow-up, criteria used and time of interim PET. In conclusion, the use of a PET-positive study as a surrogate marker was hampered by inconsistent interpretation criteria and study populations. However, the high negative predictive value may permit treatment stratification based on a negative outcome.

\section{$9.4 \quad{ }^{18}$ F-FDG PET or PET/CT in Prognosis/Outcome Evaluation}

\subsubsection{Hodgkin and Non-Hodgkin Lymphomas}

Wang et al. [13] carried out a meta-analysis to detect the prognostic power of ${ }^{18} \mathrm{~F}-\mathrm{FDG}$ PET in the evaluation of pre-stem cell transplantation (SCT) and post-SCT in HL and NHL. For the
pre-SCT PET or PET/CT, the combined hazard ratios (HRs) of PET for progression-free survival (PFS) and overall survival (OS) were 2.32 and 2.64, respectively. Subgroup analysis showed that the HRs of PFS for HL and NHL were 3.28 and 2.0, respectively. For the post-SCT PET scan, the combined HR for PFS was 4.61. The authors found that ${ }^{18} \mathrm{~F}$-FDG PET was especially effective in predicting pre-STC and post-STC prognosis. The patients with a negative PET scan had a better prognosis compared with those with a positive scan for PFS and OS. In the subgroup analysis, ${ }^{18}$ F-FDG PET had a higher value in predicting prognosis before SCT for HL patients.

Burggraaff et al. [14] aimed to assess the predictive value of visually assessed interim ${ }^{18} \mathrm{~F}$ FDG PET on PFS or event-free survival (EFS) in DLBCL patients treated with first-line immunochemotherapy regimens. The pooled HR was $3.13(95 \%$ CI $2.52-3.89)$ with a $95 \%$ prediction interval of $1.68-5.83$. The negative predictive value for progression generally exceeded $80 \%$, but sensitivity, specificity and positive predictive values ranged widely. These findings showed that interim ${ }^{18} \mathrm{~F}$-FDG PET has predictive value in DLBCL patients. Some diagnostic test characteristics were not satisfactory, especially the positive predictive value should be improved before a successful risk stratified treatment approach can be implemented in clinical practice.

Adams et al. [15] systematically reviewed and meta-analysed the prognostic value of complete remission status at end-of-treatment ${ }^{18} \mathrm{~F}-\mathrm{FDG}$ PET in DLBCL patients treated with R-CHOP. The disease relapse rate among all patients with complete remission status according to end-of-treatment ${ }^{18} \mathrm{~F}$-FDG PET ranged from 7 to $20 \%$, with a weighted summary proportion of $13.7 \%$. In conclusion, a non-negligible proportion of R-CHOP-treated DLBCL patients who achieve complete remission according to end-of-treatment ${ }^{18} \mathrm{~F}$-FDG PET experiences disease relapse during follow-up.

Adams et al. [16] analysed the prognostic value of interim ${ }^{18} \mathrm{~F}$-FDG PET in DLBCL patients treated with R-CHOP. At multivariable analysis, two studies reported interim ${ }^{18} \mathrm{~F}-\mathrm{FDG}$ PET to have independent prognostic value in addition to the International Prognostic Index (IPI) in pre- 
dicting treatment failure, whereas three studies reported that this was not the case. One study reported interim 18F-FDG PET to have independent prognostic value in addition to the IPI in predicting death, whereas two studies reported that this was not the case. In conclusion, interim ${ }^{18} \mathrm{~F}$-FDG-PET in R-CHOP-treated DLBCL has some correlation with outcome, but its prognostic value is homogeneously sub-optimal across studies and it has not consistently proven to surpass the prognostic potential of the IPI. Therefore, at present there is no scientific base to support the clinical use of interim ${ }^{18} \mathrm{~F}$-FDG-PET in R-CHOPtreated DLBCL.

Zhu et al. [17] analysed the prognostic value of interim ${ }^{18} \mathrm{~F}$-FDG PET in DLBCL patients treated with rituximab-based immunochemotherapy. The pooled HR comparing PFS between patients with positive and negative results was $2.96(95 \%$ CI $=2.25-3.89)$. The patients in interim ${ }^{18} \mathrm{~F}-\mathrm{FDG}$ PET-negative group had a higher complete response (CR) rates than those in interim ${ }^{18} \mathrm{~F}$-FDG PET-positive group (relative risk $=5.53,95 \% \mathrm{CI}=2.59-11.8$ ). The authors concluded that consistent evidence favouring interim ${ }^{18} \mathrm{~F}$-FDG PET-based treatment assessment should be considered in the management of patients with DLBCL.

Pyo et al. [18] evaluated post-chemotherapy response assessment in FL. The pooled HR of end-therapy ${ }^{18} \mathrm{~F}$-FDG PET and CT were 5.1 (95\%CI: 3.7-7.2) and 2.6 (95\%CI: 1.2-5.8), respectively, which implies that PET is more predictive of PFS after chemotherapy than CT. The pooled CR rates of PET- and CT-based response criteria were $75 \%$ (95\% CI: $70-79 \%$ ) and $63 \%$ (95\%CI: 53-73\%), respectively, which implies that PET is more efficient in distinguishing CR from other states with residual disease. The authors concluded that PET-based treatment assessment should be considered in the management of patients with FL.

Liao et al. [19] evaluated the prognostic value of ${ }^{18} \mathrm{~F}$-FDG PET/CT visual interpretation in patients with aggressive NHL. PFS and OS of PET/CT-positive patients were significantly lower when determined by the visual method. In subgroup analysis, International Harmonization Project (IHP), Deauville criteria, and having no standard interpretation groups were factors able to predict PFS; IHP and having no standard interpretation group were able to predict OS. With PET/CT, IHP and Deauville 5-point criteria, the PFS of patients receiving 2-4 cycles of chemotherapy before PET/CT was significantly lower than that of PET/CT-negative patients. No significant difference in OS was observed when patients received 3 or fewer cycles of chemotherapy before PET/CT, though OS was significantly lower in patients receiving more than 3 chemotherapy cycles. They concluded that interim PET/ CT analysis after 3-4 chemotherapy cycles is capable of predicting disease prognosis in aggressive NHL.

Adams et al. [20] aimed to analyse the value of pretransplant ${ }^{18} \mathrm{~F}$-FDG PET in predicting outcome after autologous stem cell transplantation in aggressive NHL. Pooled sensitivity and specificity of ${ }^{18} \mathrm{~F}$-FDG PET were 54 and $73.1 \%$ in predicting treatment failure, and 54.5 and $68.7 \%$ in predicting death. They concluded that pretransplant ${ }^{18} \mathrm{~F}$-FDG PET cannot be recommended in aggressive NHL, because available studies suffer from major methodological flaws, and reported prognostic estimates are low.

Zhu et al. [21] aimed to determine the prognostic value of interim and final ${ }^{18} \mathrm{~F}$-FDG PET in NHL patients treated with rituximab-containing chemotherapy. The combined HRs of interim PET for PFS and OS in DLBCL were 4.4 $(p=0.11)$ and $3.99(p=0.46)$, respectively. The combined HRs of final PET for PFS and OS in DLBCL were $5.91(p=0.39)$ and $6.75(p=0.92)$, respectively. Regarding non-DLBCL with final PET, the combined HRs of final PET for PFS and OS were $4.05(p=0.79)$ and $5.1(p=0.51)$, respectively. In conclusion, in DLBCL, both interim and final PET can be performed for survival and progression analysis. But in other NHL, it would be necessary to perform final PET for predictive purposes.

Adams et al. [22] aimed to systematically review and meta-analyse the value of interim ${ }^{18} \mathrm{~F}-$ FDG PET in predicting treatment failure in HL. The area under the summary ROC curve was 0.877. Pooled sensitivity and specificity were 70.8\% (95\%CI: 64.7-76.4\%) and 89.9\% (95\%CI: $88-91.6 \%)$. The overall prognostic value of 
interim PET appeared to be moderate for excluding and relatively high for identifying treatment failure in HL. However, they stated that interim PET cannot yet be implemented in routine clinical practice due to moderate-quality evidence and inter-study heterogeneity that cannot be fully explained yet.

Sickinger et al. [23, 24] assessed the effects of interim ${ }^{18} \mathrm{~F}$-FDG PET treatment modification in individuals with HL. PFS was shorter in participants with PET-adapted therapy (without radiotherapy) than in those receiving standard treatment with radiotherapy (HR: 2.38; 95\%CI: 1.62-3.5). This difference was also apparent in comparisons of participants receiving no additional radiotherapy (PET-adapted therapy) versus radiotherapy (HR: 1.86 (95\%CI: 1.07-3.23) and in those receiving chemotherapy but no radiotherapy (PET-adapted therapy) versus standard radiotherapy (HR: 3.0; 95\%CI: 1.75-5.14). Overall, this systematic review found moderatequality evidence that PFS was shorter in individuals with early-stage HL and a negative PET receiving chemotherapy only (PET-adapted therapy) than in those receiving additional radiotherapy (standard therapy). It was still uncertain whether PET-positive individuals benefit from PET-based treatment adaptation and the effect of such an approach in those with advanced HL.

Adams et al. [25] aimed to systematically review the prognostic value of pretransplant ${ }^{18} \mathrm{~F}-$ FDG PET in refractory/relapsed HL treated with autologous stem cell transplantation (SCT). Pooled sensitivity and specificity of pretransplant ${ }^{18} \mathrm{~F}-\mathrm{FDG}$ PET in predicting treatment failure (i.e. either progressive, residual, or relapsed disease) were $67.2 \%$ (95\%CI: $58.2-75.3 \%$ ) and $70.7 \%$ (95\%CI: 64.2-76.5\%), respectively. Pooled sensitivity and specificity of pretransplant ${ }^{18} \mathrm{~F}-\mathrm{FDG}$ PET in predicting death during follow-up were $74.4 \%$ (95\%CI: $58.8-86.5 \%$ ) and 58\% (95\%CI: $49.3-66.3 \%$ ), respectively. In conclusion, the moderate quality of evidence suggested pretransplant ${ }^{18} \mathrm{~F}$-FDG-PET to have value in predicting outcome in refractory/relapsed HL patients treated with autologous SCT. Nevertheless, a considerable proportion of pretransplant ${ }^{18} \mathrm{~F}-$ FDG PET-positive patients remained disease free and a considerable proportion of pretransplant ${ }^{18}$ F-FDG PET-negative patients developed disease relapse after autologous SCT.

Adams et al. [26] systematically reviewed and meta-analysed the outcome of HL patients with a post-treatment ${ }^{18} \mathrm{~F}$-FDG PET-negative residual mass. The disease relapse rate in HL patients with a ${ }^{18} \mathrm{~F}$-FDG PET-negative residual mass after first-line therapy was approximately $6.8 \%$. They concluded that the presence of a non- ${ }^{18} \mathrm{~F}-\mathrm{FDG}-$ avid residual mass has not been proven yet to be associated with a worse outcome than a posttreatment ${ }^{18} \mathrm{~F}$-FDG-PET-based complete remission status without a residual mass.

The same group [27] analysed the prognostic value of complete remission status at ${ }^{18} \mathrm{~F}-\mathrm{FDG}$ PET in HL after completion of first-line therapy. The pooled disease relapse rate during follow-up among all patients with complete remission status at end-of-treatment ${ }^{18} \mathrm{~F}$-FDG-PET was $7.5 \%$ (95\%CI: $3.9-13.8 \%$ ). They concluded that, although the disease relapse rate in HL patients who achieve an ${ }^{18} \mathrm{~F}$-FDG PET-based complete remission after first-line therapy is low, it is actually high when considering the generally favourable outcome of HL.

\subsubsection{Multiple Myeloma}

Caldarella et al. [28] aimed to evaluate the usefulness of ${ }^{18} \mathrm{~F}-\mathrm{FDG}$ PET or PET/CT in monitoring response to treatment in patients with MM. Based on the findings from the literature, ${ }^{18} \mathrm{~F}-\mathrm{FDG}$ PET or PET/CT appeared to be useful in the assessment of treatment response in patients with MM. In particular, PET or PET/CT could detect the response to treatment earlier than other imaging. Negative findings on posttreatment ${ }^{18} \mathrm{~F}$-FDG PET or PET/CT were mostly correlated with complete clinical and histological remission or, at least, low risk of recurrences or disease progression. Persistence of metabolically active lesions was related to shorter overall and event-free survival. Therefore, post-treatment ${ }^{18}$ F-FDG PET findings could be of higher prognostic significance than standard response monitoring methods. In the near future, ${ }^{18} \mathrm{~F}$-FDG PET 
or PET/CT will be used even more in the assessment of metabolic response after treatment in patients with MM, as a guidance for clinical decision and to eventually decide for alternative therapies in non-responding patients.

\subsection{Prognostic Role of Semi- quantitative PET Parameters}

Guo et al. [29] have analysed whether baseline metabolic tumour volume (MTV) and total lesion glycolysis (TLG) measured by ${ }^{18} \mathrm{~F}$-FDG PET/CT affect prognosis of patients with lymphoma. Patients with high baseline MTV showed a worse prognosis considering PFS and OS as well as patients with high baseline TLG. A high baseline MTV was significantly associated with worse survival in DLBCL patients treated with R-CHOP as well as a high baseline TLG. The negative effect of high baseline MTV on PFS was demonstrated in HL. A high baseline MTV was significantly associated with worse survival in ENKTL patients. A high baseline TLG was significantly associated with worse survival in ENKL patients. The authors concluded that high baseline MTV or TLG predict significantly worse PFS and OS in patients with lymphoma.

Wang et al. [30] evaluated the prognostic value of maximum standardized uptake value (SUVmax), MTV, and TLG of baseline, interim and end-of-treatment ${ }^{18} \mathrm{~F}$-FDG PET/CT parameters in ENKTL. SUVmax, MTV and TLG on baseline PET/CT were significantly associated with PFS and with OS. For the delta SUV (DS) on interim PET/CT, the HRs for PFS and OS were 5.15 (95\% CI 2.71-9.80) and 5.8 (95\%CI 2.28-14.73), respectively. Similarly, the DS on end-of-treatment PET/CT was a significant predictor of PFS and OS with HRs of 3.65 (95\%CI: 2.13-6.26) and 3.32 (95\%CI: 1.79-6.15), respectively. They suggested that SUVmax, MTV, TLG on baseline PET/CT, DS on interim PET/CT and DS on end-of-treatment PET/CT may be significant prognostic indicators for PFS and OS in ENKTL patients. Moreover, TLG tended to be superior to SUVmax and MTV on baseline PET/ CT for predicting survival of ENKTL patients.
Therefore, response monitoring and prognostication assessments based on multiple PET/CT parameters should be considered in the management of ENKTL patients.

Xie et al. [31] analysed whether SUVmax, MTV and TLG acquired from ${ }^{18} \mathrm{~F}$-FDG PET/CT are predictors of prognosis of DLBCL. Combined results suggested a strong link between the high SUVmax, MTV and TLG values and the poor 3-year PFS with ORs of 2.59, 3.69 and 2.29, respectively. Similarly, high MTV and TLG values unfavourably influenced the 3-year OS with ORs of 5.40 and 2.19, respectively. The pooled results also showed that high SUVmax and MTV were negative predictors of PFS. The TLG value was not predictive of PFS. And for OS, only high MTV was a strong predictor of poor prognosis in DLBCL. Their results suggested that SUVmax and MTV may be significant prognostic markers for PFS and MTV may be the only predictor for OS in DLBCL.

\section{$9.6{ }^{18}$ F-FDG PET or PET/CT in Comparison with Magnetic Resonance Imaging}

Wang et al. [32] aimed to compare the performance of whole-body magnetic resonance imaging (WB-MRI) with that of ${ }^{18} \mathrm{~F}$-FDG PET/CT for lesion detection and initial staging in patients with aggressive or indolent lymphoma. In terms of staging, the pooled accuracy of WB-MRI and ${ }^{18} \mathrm{~F}-\mathrm{FDG}$ PET/CT for HL and aggressive NHL were 98\% (95\%CI: $94-100 \%)$ and 98\% (95\%CI: 94-100\%), respectively. The pooled accuracy of ${ }^{18} \mathrm{~F}-\mathrm{FDG}$ PET/CT dropped to $87 \%$ (95\% CI: 72-97\%) for staging in patients with indolent lymphoma, whereas that of WB-MRI remained 96\% (95\%CI: 91-100\%). Subgroup analysis indicated an even lower accuracy of ${ }^{18} \mathrm{~F}-\mathrm{FDG}$ PET/CT for staging of less ${ }^{18} \mathrm{~F}-\mathrm{FDG}$-avid indolent NHLs (60\%; 95\% CI: 23-92\%), in contrast to the superior performance of WB-MRI (98\%; 95\%CI: $88-100 \%)$. The authors concluded that WB-MRI is a promising radiation-free imaging technique that may serve as a viable alternative to 
${ }^{18}$ F-FDG PET/CT for staging of ${ }^{18} \mathrm{~F}-\mathrm{FDG}$-avid lymphomas, where ${ }^{18} \mathrm{~F}$-FDG $\mathrm{PET} / \mathrm{CT}$ remains the standard of care. Additionally, WB-MRI seemed a less histology-dependent functional imaging test than ${ }^{18} \mathrm{~F}-\mathrm{FDG}$ PET/CT and may be the imaging test of choice for staging of indolent NHLs with low ${ }^{18}$ F-FDG avidity.

Regacini et al. [33] aimed to compare WB-MRI with ${ }^{18} \mathrm{~F}$-FDG PET/CT for lymphoma staging. WB-MRI and ${ }^{18} \mathrm{~F}-\mathrm{FDG}-\mathrm{PET} / \mathrm{CT}$ agreed in $90.5 \%$ of the cases. In most of the studies, when there was disagreement between the methods, WB-MRI overstaged in relation to ${ }^{18} \mathrm{~F}-\mathrm{FDG}$ PET/CT. The sensitivity of WB-MRI and ${ }^{18} \mathrm{~F}-\mathrm{FDG}$ PET/CT, in comparison with the clinical-radiological standard, ranged from 59 to $100 \%$ and from 63 to $100 \%$, respectively. The authors concluded that WB-MRI has excellent agreement with ${ }^{18} \mathrm{~F}-\mathrm{FDG}$ $\mathrm{PET} / \mathrm{CT}$ and is a great alternative for managing lymphoma patients, without using ionizing radiation or an intravenous contrast agent.

Gariani et al. [34] evaluated the diagnostic performance of WB-MRI including diffusion weighted sequences (DWI) compared to wholebody $\mathrm{CT}$ or ${ }^{18} \mathrm{~F}-\mathrm{FDG} \mathrm{PET} / \mathrm{CT}$ in patients with MM. WB-MRI detected more lesions than ${ }^{18} \mathrm{~F}-$ FDG PET/CT (sensitivity 68-100\% versus 47-100\%) but was less specific (specificity $37-83 \%$ versus 62-85.7\%). Despite these insights the authors concluded that, because of the heterogeneity of the studies, future prospective trials should assess the impact of WB-MRI on management of MM.

Weng et al. [35] conducted a systematic review of the published literature to evaluate the diagnostic accuracy of ${ }^{18} \mathrm{~F}$-FDG PET, ${ }^{18} \mathrm{~F}$-FDG $\mathrm{PET} / \mathrm{CT}, \mathrm{MRI}$ and scintigraphy for MM-related bone disease. For ${ }^{18} \mathrm{~F}$-FDG PET and PET/CT, pooled sensitivity and specificity were $91 \%$ and $69 \%$, respectively. Statistically significant differences were not found in the sensitivity and specificity between MRI, scintigraphy, ${ }^{18}$ F-FDG-PET and PET/CT. In conclusion, the authors suggested that ${ }^{18} \mathrm{~F}-\mathrm{FDG}-\mathrm{PET}$, PET/CT, MRI and scintigraphy are all associated with high detection rate of bone disease in patients with MM. Thus, in clinical practice, it is recommended that we could choose these tests according to the condition of the patient.

\subsection{Conclusions}

Overall, ${ }^{18} \mathrm{~F}-\mathrm{FDG}$ PET or PET/CT appears to be a useful and accurate diagnostic tool for haematological malignancies in clinical practice from an "evidence-based" point of view. Some topics and results need further investigations in order to overcome methodological limits and clarify the real diagnostic role of this tool and its more appropriate position in the diagnostic flow chart.

\section{References}

1. Montes de Jesus FM, Kwee TC, Nijland M, Kahle XU, Huls G, Dierckx RAJO, et al. Performance of advanced imaging modalities at diagnosis and treatment response evaluation of patients with posttransplant lymphoproliferative disorder: a systematic review and meta-analysis. Crit Rev Oncol Hematol. 2018;132:27-38.

2. Adams HJ, Nievelstein RA, Kwee TC. Systematic review on the additional value of $18 \mathrm{~F}$-fluoro-2-deoxyD-glucose positron emission tomography in staging follicular lymphoma. J Comput Assist Tomogr. 2017;41:98-103.

3. Treglia G, Zucca E, Sadeghi R, Cavalli F, Giovanella L, Ceriani L. Detection rate of fluorine-18fluorodeoxyglucose positron emission tomography in patients with marginal zone lymphoma of MALT type: a meta-analysis. Hematol Oncol. 2015;33:113-24.

4. Adams HJ, Kwee TC, de Keizer B, Fijnheer R, de Klerk JM, Nievelstein RA. FDG PET/CT for the detection of bone marrow involvement in diffuse large B-cell lymphoma: systematic review and meta-analysis. Eur J Nucl Med Mol Imaging. 2014;41:565-74.

5. Adams HJ, Kwee TC, de Keizer B, Fijnheer R, de Klerk JM, Littooij AS, et al. Systematic review and meta-analysis on the diagnostic performance of FDG$\mathrm{PET} / \mathrm{CT}$ in detecting bone marrow involvement in newly diagnosed Hodgkin lymphoma: is bone marrow biopsy still necessary? Ann Oncol. 2014;25:921-7.

6. Cheng G, Alavi A. Value of 18F-FDG PET versus iliac biopsy in the initial evaluation of bone marrow infiltration in the case of Hodgkin's disease: a metaanalysis. Nucl Med Commun. 2013;34:25-31.

7. Ji J, Liu XH, She NN, Li L, Zhang XB. Value of 18 FFDG PET/CT and PET in diagnosing and staging extranodal nasal type natural killer/T-cell lymphoma: a meta-analysis. Lin Chung Er Bi Yan Hou Tou Jing Wai Ke Za Zhi. 2018;32:1876-82.

8. Zhou X, Lu K, Geng L, Li X, Jiang Y, Wang X. Utility of PET/CT in the diagnosis and staging of extranodal natural killer/T-cell lymphoma: a systematic review and meta-analysis. Medicine. 2014;93:e258.

9. Lu YY, Chen JH, Lin WY, Liang JA, Wang HY, Tsai $\mathrm{SC}$, et al. FDG PET or PET/CT for detecting intra- 
medullary and extramedullary lesions in multiple myeloma: a systematic review and meta-analysis. Clin Nucl Med. 2012;37:833-7.

10. Adams HJA, Kwee TC. Proportion of false-positive lesions at interim and end-of-treatment FDG-PET in lymphoma as determined by histology: systematic review and meta-analysis. Eur J Radiol. 2016;85:1963-70.

11. Sun N, Zhao J, Qiao W, Wang T. Predictive value of interim PET/CT in DLBCL treated with R-CHOP: meta-analysis. Biomed Res Int. 2015;2015:648572.

12. Ziakas PD, Poulou LS, Voulgarelis M, Thanos L. The Gordian knot of interim 18-fluorodeoxyglucose positron emission tomography for Hodgkin lymphoma: a meta-analysis and commentary on published studies. Leuk Lymphoma. 2012;53:2166-74.

13. Wang C, Li P, Wu S, Lu J, Liu Q, Luo H, et al. The role of fluorine-18 fluorodeoxyglucose PET in prognosis evaluation for stem cell transplantation of lymphoma: a systematic review and meta-analysis. Nucl Med Commun. 2016;37:338-47.

14. Burggraaff $\mathrm{CN}$, de Jong A, Hoekstra OS, Hoetjes NJ, Nievelstein RAJ, Jansma EP, et al. Predictive value of interim positron emission tomography in diffuse large B-cell lymphoma: a systematic review and meta-analysis. Eur J Nucl Med Mol Imaging. 2019;46:65-79.

15. Adams HJ, Nievelstein RA, Kwee TC. Prognostic value of complete remission status at end-of-treatment FDG-PET in R-CHOP-treated diffuse large B-cell lymphoma: systematic review and meta-analysis. $\mathrm{Br}$ J Haematol. 2015;170:185-91.

16. Adams HJ, Kwee TC. Prognostic value of interim FDG-PET in R-CHOP-treated diffuse large B-cell lymphoma: systematic review and meta-analysis. Crit Rev Oncol Hematol. 2016;106:55-63.

17. Zhu D, Xu XL, Fang C, Ji M, Wu J, Wu CP, et al. Prognostic value of interim (18)F-FDG-PET in diffuse large B cell lymphoma treated with rituximab-based immune-chemotherapy: a systematic review and meta-analysis. Int J Clin Exp Med. 2015;8:15340-50.

18. Pyo J, Won Kim K, Jacene HA, Sakellis CG, Brown JR, Van den Abbeele AD. End-therapy positron emission tomography for treatment response assessment in follicular lymphoma: a systematic review and metaanalysis. Clin Cancer Res. 2013;19:6566-77.

19. Liao CC, Qin YY, Tan XH, Hu JJ, Tang Q, Rong Y, et al. Predictive value of interim PET/CT visual interpretation in the prognosis of patients with aggressive non-Hodgkin's lymphoma. Onco Targets Ther. 2017;10:5727-38.

20. Adams HJ, Kwee TC. Pretransplant FDG-PET in aggressive non-Hodgkin lymphoma: systematic review and meta-analysis. Eur J Haematol. 2017;98:337-47.

21. Zhu Y, Lu J, Wei X, Song S, Huang G. The predictive value of interim and final $[18 \mathrm{~F}]$ fluorodeoxyglucose positron emission tomography after rituximabchemotherapy in the treatment of non-Hodgkin's lymphoma: a meta-analysis. Biomed Res Int. 2013;2013:275805.
22. Adams HJ, Nievelstein RA, Kwee TC. Prognostic value of interim FDG-PET in Hodgkin lymphoma: systematic review and meta-analysis. Br J Haematol. 2015;170:356-66.

23. Sickinger MT, von Tresckow B, Kobe C, Engert A, Borchmann P, Skoetz N. Positron emission tomography-adapted therapy for first-line treatment in individuals with Hodgkin lymphoma. Cochrane Database Syst Rev. 2015;1:CD010533.

24. Sickinger MT, von Tresckow B, Kobe C, Borchmann P, Engert A, Skoetz N. PET-adapted omission of radiotherapy in early stage Hodgkin lymphoma-a systematic review and meta-analysis. Crit Rev Oncol Hematol. 2016;101:86-92.

25. Adams HJ, Kwee TC. Prognostic value of pretransplant FDG-PET in refractory/relapsed Hodgkin lymphoma treated with autologous stem cell transplantation: systematic review and meta-analysis. Ann Hematol. 2016;95:695-706.

26. Adams HJ, Nievelstein RA, Kwee TC. Outcome of Hodgkin lymphoma patients with a posttreatment 18F-fluoro-2-deoxy-d-glucose positron emission tomography (FDG-PET)-negative residual mass: systematic review and meta-analysis. Pediatr Hematol Oncol. 2015;32:515-24.

27. Adams HJ, Nievelstein RA, Kwee TC. Systematic review and meta-analysis on the prognostic value of complete remission status at FDG-PET in Hodgkin lymphoma after completion of first-line therapy. Ann Hematol. 2016;95:1-9.

28. Caldarella C, Isgrò MA, Treglia I, Treglia G. Is fluorine-18-fluorodeoxyglucose positron emission tomography useful in monitoring the response to treatment in patients with multiple myeloma? Int J Hematol. 2012;96:685-91.

29. Guo B, Tan X, Ke Q, Cen H. Prognostic value of baseline metabolic tumor volume and total lesion glycolysis in patients with lymphoma: a meta-analysis. PLoS One. 2019; 14:e0210224.

30. Wang H, Shen G, Jiang C, Li L, Cui F, Tian R. Prognostic value of baseline, interim and end-oftreatment $18 \mathrm{~F}-\mathrm{FDG}$ PET/CT parameters in extranodal natural killer/T-cell lymphoma: a meta-analysis. PLoS One. 2018;13:e0194435.

31. Xie M, Wu K, Liu Y, Jiang Q, Xie Y. Predictive value of F-18 FDG PET/CT quantization parameters in diffuse large B cell lymphoma: a meta-analysis with 702 participants. Med Oncol. 2015;32:446.

32. Wang D, Huo Y, Chen S, Wang H, Ding Y, Zhu X, et al. Whole-body MRI versus $18 \mathrm{~F}-F D G$ PET/CT for pretherapeutic assessment and staging of lymphoma: a meta-analysis. Onco Targets Ther. 2018;11:3597-608.

33. Regacini R, Puchnick A, Shigueoka DC, Iared W, Lederman HM. Whole-body diffusion-weighted magnetic resonance imaging versus FDG-PET/CT for initial lymphoma staging: systematic review on diagnostic test accuracy studies. Sao Paulo Med J. 2015;133:141-50.

34. Gariani J, Westerland O, Natas S, Verma H, Cook G, Goh V. Comparison of whole body magnetic resonance imaging (WBMRI) to whole body computed 
tomography (WBCT) or 18F-fluorodeoxyglucose positron emission tomography/CT (18F-FDG PET/ $\mathrm{CT}$ ) in patients with myeloma: systematic review of diagnostic performance. Crit Rev Oncol Hematol. 2018;124:66-72.
35. Weng WW, Dong MJ, Zhang J, Yang J, Xu Q, Zhu YJ, et al. A systematic review of MRI, scintigraphy, FDGPET and PET/CT for diagnosis of multiple myeloma related bone disease-which is best? Asian Pac J Cancer Prev. 2014;15:9879-84.

Open Access This chapter is licensed under the terms of the Creative Commons Attribution 4.0 International License (http://creativecommons.org/licenses/by/4.0/), which permits use, sharing, adaptation, distribution and reproduction in any medium or format, as long as you give appropriate credit to the original author(s) and the source, provide a link to the Creative Commons license and indicate if changes were made.

The images or other third party material in this chapter are included in the chapter's Creative Commons license, unless indicated otherwise in a credit line to the material. If material is not included in the chapter's Creative Commons license and your intended use is not permitted by statutory regulation or exceeds the permitted use, you will need to obtain permission directly from the copyright holder. 